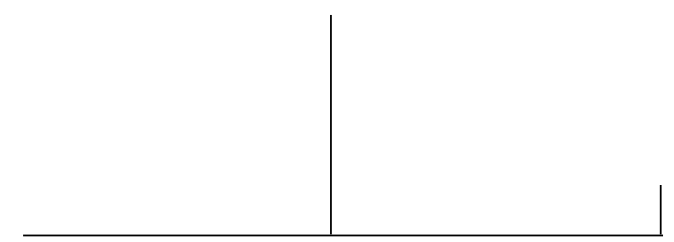

Rev. Latinoam. Psicopat. Fund., São Paulo, v. 15, n. 1, p. 171-196, março 2012

\title{
Psicopatologia descritiva: aspectos históricos e conceituais*
}

German E. Berrios

Este artigo oferece uma análise conceitual e histórica da psicopatologia descritiva. A primeira seção a define como um sistema cognitivo constituído por termos, suposições e regras para a sua aplicação. Traça as implicações conceituais dessa definição e as relaciona à prática clínica. A segunda seção contém uma avaliação do trabalho histórico sobre a psicopatologia descritiva e oferece uma hipótese para explicar o seu desenvolvimento durante o século XIX. Sugere-se que o trabalho envolvido no teste de hipóteses deve ser realizado por psiquiatras com formação em história e enquanto uma especialidade separada. Conclui-se que a calibração histórica dos sintomas psiquiátricos deve ser considerada como uma etapa essencial na construção de uma psicopatologia descritiva viável.

Palavras-chave: Psicopatologia - história, modelos psicológicos, psiquiatria - história, linguagem

* Publicação original: Descriptive psychopathology: conceptual and historical aspects, Psychological Medicine, n. 14, p. 303-313, 1984.

Tradução de Lazslo A. Ávila e revisão técnica de Ana Maria G. R. Oda. 


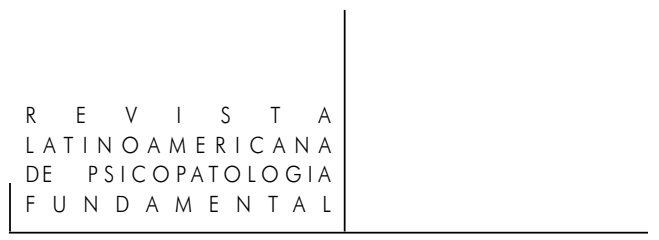

\section{Introdução}

O temor de que a história da medicina e da psiquiatria possa tornar-se subserviente aos médicos levou alguns historiadores a superestimar sua autonomia. Em relação à psiquiatria, uma expressão comum dessa preocupação tem sido a de sobre-enfatizar os seus aspectos sociais (Dörner, 1969). Em relação à medicina em geral alguns se sentem autorizados a escrever: "O compromisso com uma história médica 'neutra' não foi apenas difícil, mas muitas vezes pôde gerar nada menos do que má história - uma história com a raiva deixada de fora" (Neve, 1983).

Embora haja algo a ser dito em favor de escrever a história "raivosa", principalmente em relação aos aspectos sociopolíticos da psiquiatria, a mudança sugerida também teve indesejáveis consequências. A preocupação exclusiva com os macroconceitos e as questões sociais têm, em certas ocasiões, resultado em obras psiquiátricas históricas que se lêem como manifestos políticos (Castel, 1973; Dörner, 1969; Blasius, 1980; Scull, 1979).

Uma vítima deste modismo tem sido a investigação histórica sobre a origem e evolução dos sintomas psiquiátricos. A história da psicopatologia descritiva, devido à sua especial vulnerabilidade às acusações de "presentismo", ou "internalismo" foi deixada para os clínicos idosos ou confinada às seções introdutórias de estudos clínicos.

Há uma demanda, no entanto, para este tipo de estudo. As dores éticas dos anos 1960, provocadas pelo movimento da antipsiquiatria, foram substituídas pelo furor descritivo dos anos 1970 . 


\begin{tabular}{c|c} 
HISTÓRIA DA \\
PSICOPATOLOGIA, \\
POR GERMAN E. \\
BERRIOS \\
\hline
\end{tabular}

Aqueles autores, cuja tarefa é a calibração da nomenclatura da psiquiatria, necessitam da ajuda dos historiadores. A resposta entusiástica destes últimos, no entanto, levou [os psiquiatras] a uma fabricação histórica de "faça-você-mesmo", que luta sem sucesso contra a falta de treino formal histórico, acesso limitado a fontes primárias e obstáculos linguísticos (Andreasen, 1982; Rome, 1979; Munro, 1982; Kendler \& Tsuang, 1981). Como consequência, os padrões de pesquisa na história psiquiátrica, que deveriam ser tão rigorosos quanto em neuroquímica, não foram mantidos.

Este trabalho apresenta a visão de que o trabalho colaborativo entre os médicos psiquiatras e os historiadores é urgentemente necessário e sugere, como primeira tarefa comum, a calibração da psicopatologia descritiva. Como um passo inicial para esta aproximação, oferece uma análise da estrutura conceitual da psicopatologia descritiva e então descreve um modelo geral para explicar a sua gênese durante o século XIX. Este modelo pode ser empiricamente testado através da realização, por exemplo, de estudos sobre a evolução dos sintomas individuais (Berrios, 1977, 1981a, b, 1982).

\section{Questões conceituais}

A psiquiatria e sua linguagem

A psiquiatria é definida neste trabalho como o conjunto de consequências (cognitivas e não cognitivas) que resultam da utilização adequada de uma linguagem psicopatológica. "Cognitivo" deve ser entendido como "científico" ou clínico e "não cognitivo" significa "sociopolítico" (McLeod, 1977).

Uma linguagem psicopatológica, por sua vez, é definida como uma coleção sistemática de princípios gerais, enunciados descritivos, e regras para a sua aplicação. Discursos regionais deste tipo foram criados pela maioria dos grupos sociais para descrever as formas de comportamento assumidos como resultantes de disfunções corporais ou psicológicas indesejáveis (Miller \& Berrios, 1984).

A utilização da linguagem psicopatológica é considerada como "apropriada" quando é limitada ao domínio onde foi calibrada. A calibração da linguagem baseia-se na observação histórica, clínica e estatística cumulativa. Uma vez atingido um "estado de equilíbrio" [steady state], a linguagem clínica perdura até que (1) os objetivos cognitivos da "comunidade de usuários" (Laudan, 1977) ou "coletivo de pensamento" (Fleck, 1979) mudem de direção, ou (2) o objeto psicopatológico em si passe por uma transformação para além dos limites permissíveis (Hare, 1974; Marchais, 1983), ou (3) a interação controlada entre o objeto e a lin- 
guagem promova maior desenvolvimento científico (Pandit, 1983). A psicopatologia descritiva deve ser considerada como uma instituição conceitual que oferece uma interface dinâmica entre o observador e os fenômenos psicopatológicos.

\section{Fenômenos psicopatológicos e o observador}

O comportamento psicopatológico consiste em um caleidoscópico conglomerado de sintomas de configuração e duração variáveis. Em essência, estes sintomas não são mais do que variações identificáveis na forma e no conteúdo do discurso e nos padrões de motilidade do paciente. Por isso, uma pura descrição "fenomenológica" dessas variações é raramente (ou nunca) alcançada na prática clínica; a situação mais comum é de interpretação superficial em termos de sistemas compartilhados de pistas sociais. Sintomas psicopatológicos têm, portanto, dois componentes: uma fonte biológica (uma disfunção), que engendra um deslocamento do comportamento, e um aspecto psicossocial que diz respeito à interpretação do deslocamento comportamental pelo próprio paciente e pelos demais. O "ruído" psicossocial, associado a cada sintoma, pode variar de estados em que ele é muito acentuado, tal como nos sintomas de interação como a manipulação (Mackenzie et al., 1978) até disfunções comportamentais que são manifestações estereotipadas de disfunção de estrutura (hardware) como a própria desorientação (Berrios, 1983).

A observação clínica do fenômeno psicopatológico é controlada por um conjunto perceptual (Blashfield et al., 1982; Sülz \& Gigerenzer, 1982). A inevitabilidade deste viés de observação vem exercitando os filósofos da ciência há muito tempo (Hesse, 1966; Wartofsky, 1979). Outra fonte de viés do observador ou instabilidade diz respeito à ocorrência de periódicas mudanças "epistemológicas" (Bachelard, 1938; Marchais, 1983).

Esta dependência da psicopatologia descritiva sobre o controle da themata (Holton, 1973) propiciou interpretações "relativistas" e a crença errônea de que os sintomas seriam apenas construções "culturais". Variações relacionadas com a cultura na apresentação de um sintoma, no entanto, não significam necessariamente que não haja nenhuma base biológica para ele ou que, se houver, seja irrelevante para o seu entendimento (Rack, 1982). Paran-Vial (1966) mostrou que os fatos de observação nas ciências comportamentais são, apesar do ruído social, "parcialmente intercambiáveis". Esta "constância" deveria tornar possível para os médicos "triangular" o sintoma e autenticar qualquer excesso cultural que possa estar mascarando a disfunção subjacente. Na verdade, esta é a fundação epistemológica da assim chamada interconfiabilidade do avaliador.

Esta "constância" deveria explicar a surpreendente estabilidade exibida por uma série de sintomas desde o século XIX. Uma vez que é duvidoso que seja to- 


\begin{tabular}{c|c} 
HISTÓRIA DA \\
PSICOPATOLOGIA, \\
POR GERMAN E. \\
BERRIOS \\
\hline
\end{tabular}

talmente devida a fatores sociais, pode ser plausivelmente afirmado que a "fixidez" formal de muitos sintomas derive, como algumas das categorias estruturais propostas por Kant e Lévi-Strauss, de um número limitado de deslocamentos de estrutura [hardware]. Isso conferiria para alguns sintomas sua qualidade estereotipada e monótona, apesar das variações acentuadas no conteúdo e na gravidade dos mesmos.

\section{Psicopatologia descritiva}

A tarefa declarada da psicopatologia descritiva, desde o início do século XIX, tem sido a identificação de classes de atos mentais anormais. De acordo com a teoria dos signos, então vigente, um nome tinha que ser dado para cada classe (Franck, 1875b). O aparecimento das "unidades de análise" tornou-se o modelo oficial em termos do qual gerações de psiquiatras passaram a "fragmentar" o comportamento de seus pacientes em sintomas discretos. Este procedimento descritivo foi considerado menos como uma "fratura" do que uma boa "desarticulação" do comportamento anormal, pois se acreditava, com base em uma versão da ontologia dos "tipos naturais", que "articulações" existissem no fluxo de comportamento. As partes resultantes, Griesinger (1867) denominou de "distúrbios elementares de doença mental" (p. 60). Desde essa época, apenas calibrações menores na terminologia foram necessárias.

A acurácia da calibração original pode ser testada de duas maneiras. Por um lado, cada descrição deveria se aplicar à maioria dos membros de sua classe em questão (vigor extensional); isso está na base da noção de validade. Por outro, seu nome deveria evocar na mente de cada observador treinado o mesmo "sintoma", "forma" ou "imagem" (vigor intensional), esta é a essência da confiabilidade.

A utilidade da calibração também pode ser testada. A maneira mais simples é examinar a eficiência das regras que decidem se um sintoma está ou não presente. Desde o século XIX, essas regras foram baseadas em pontos de corte tácitos para a intensidade, duração e frequência dos sintomas putativos, e também sobre critérios de "plausibilidade" que ajudam a avaliar os relatos do fenômeno e sua "compatibilidade" com outros sintomas. Por exemplo, na literatura histórica podem ser encontrados casos em que a "genuinidade" de um sintoma é posta em questão, a despeito do fato de que, à primeira vista, ele preencheria todos os critérios.

Por isso deve ser assumido que o critério de "plausibilidade" esteja em operação em algum nível e que a sua eficácia depende do fato de que ele seja passado de uma geração de psiquiatras para a próxima. Sugere-se neste trabalho que regras de "rejeição" estão, portanto, contidas na gramática profunda da linguagem clínica. Da mesma forma, deve-se presumir que regras transformacionais também devam existir para que sintomas atenuados possam ser 
ampliados ou postos em foco apesar do mascaramento contextual ou do ruído "patoplástico".

Uma análise similar pode ser oferecida quanto ao critério de "compatibilidade". A psicopatologia descritiva do século XIX desenvolveu, apesar de seus múltiplos sistemas taxonômicos, combinações e hierarquias de sintomas "permitidas". Sistemas classificatórios que preservaram essas combinações foram mais propensos a sobreviver do que os que ofereciam pontos de partida radicais. É sabido, por exemplo, que uma classificação das psicoses, com base em descrições de sintomas marcadamente diferentes, foi apresentada por volta do mesmo período que a de Kraepelin (Leonhard, 1957; Burckard, 1931). A falta de sucesso do sistema de Wernicke não pode ser totalmente explicada como uma falha científica, porque nenhum estudo comparativo crucial foi realizado. É mais provável que o sistema kraepeliniano expressasse melhor quais distinções e permutações estavam implícitas na psicopatologia descritiva do século XIX.

\section{A psicopatologia descritiva como um sistema cognitivo}

Um sistema cognitivo pode ser definido como um dispositivo para organizar o conhecimento. Originalmente, o termo sistema se aplicava apenas a uma coleção de itens físicos, mas desde o século XVI, também se referiu às próprias categorias (Franck, 1875a; Rescher, 1979). O conceito de um sistema diz respeito estreitamente à "teoria da verdade como correspondência": sua validade depende do sucesso com o qual sua configuração "retrata" os objetos que ele descreve.

Desde o século XVIII uma série de traços tem sido reconhecida como caracterizando um sistema. Rescher (1979) lista a totalidade, integridade, autossuficiência, coesão, consonância, arquitetura, unidade funcional, regularidade funcional, simplicidade funcional, complementaridade mútua e eficácia funcional. Estes "parâmetros" influenciaram a construção da psicopatologia descritiva durante o século XIX.

Condillac (1947), cuja influência sobre Pinel (Riese, 1968) e sobre a psiquiatria francesa do século XIX tem sido bem atestada (Le Roy, 1937), identificou os sistemas "especulativo" e "experimental". Este último, segundo ele, gera conhecimentos sobre o mundo, que estão baseados em fatos de observação. Sua visão levou a considerar a "psicopatologia" como um "sistema cognitivo" durante o século XIX; na verdade, essa crença nunca foi completamente deixada na França, como indica um apelo recente por um méthode systèmale (Marchais, 1977).

Sistemas são entidades dinâmicas e variam em estrutura de acordo com seu assunto. Rescher (1979) afirma que: 


$\mid \begin{gathered}\text { HISTÓRIA DA } \\ \text { PSICOPATOLOGIA } \\ \text { POR GERMAN E. } \\ \text { BERRIOS }\end{gathered}$

A sistematicidade tem vis-à-vis seus componentes o caráter de um perfil (em vez de uma média). Assim como a saúde de uma pessoa é determinada por uma pluralidade de fatores constituintes, assim a sistematicidade de um corpo de conhecimento é determinada em termos de uma grande variedade de considerações separáveis embora inter-relacionadas. E haverá intercâmbios entre os vários "parâmetros de sistematicidade”. (p. 11)

É do maior interesse para o historiador identificar os "intercâmbios" [trade $o f f s$, que tiveram lugar na formação da psicopatologia descritiva do século XIX. Estes podem ter sido requeridos tanto para assegurar a sobrevivência do sistema como um todo como para cumprir com as necessidades práticas de lidar com a população heterogênea dos asilos. Por exemplo, como Rescher (1979) apontou: “... uma maior abrangência pode ameaçar a consistência ... maior conexão pode exigir a inserção de elementos desuniformes" (p. 14).

\section{Tipos de psicopatologia descritiva durante o século XIX}

Duas definições complementares de psicopatologia descritiva foram originadas ao longo do século XIX. A crença de que todos os fenômenos mórbidos poderiam ser acomodados dentro das classificações em curso das funções mentais levou a um modelo de "continuidade". Carpenter (1879) se sentiu capaz de escrever no final do século: "Pode ser afirmado, sem hesitação, que não há nada nos fenômenos psíquicos da insanidade que distinga esta condição dos estados que podem ser temporariamente induzidos em mentes saudáveis?” (p. 658). A psicopatologia, neste sentido, seria apenas um ramo da psicologia ("psicologia patológica").

Por outro lado, a compreensão gradual de que alguns sintomas bizarros não encontravam contrapartida no comportamento normal levou ao desenvolvimento de um modelo de "descontinuidade", isto é, de uma "patologia psicológica". Griesinger (1867) coloca assim: "Nós encontramos outras anomalias psicológicas no insano que não tem nada de análogo no estado de saúde" (p. 62).

A psicopatologia descritiva durante este período também atuou como uma interface entre a filosofia e a psiquiatria clínica. A nova medicina desde o início do século XIX exigiu descrições refinadas das manifestações de doença (Laín Entralgo, 1978; Ackerknecht, 1967; Foucault, 1972a); a terminologia disponível de insanidade era incapaz de fornecer essas descrições. Daí escritores como Griesinger (1875) rejeitarem os termos antigos como demasiado gerais. A linguagem começou a ser considerada como relevante não só para a descrição dos sintomas, mas também para a análise da experiência subjetiva patológica (Starobinski, 1974). Para lidar com os problemas conceituais decorrentes, uma 
teoria do conhecimento era necessária e alguns alienistas propuseram a sua própria (Falret, 1864). Isto não foi sempre aceitável para os filósofos que apontaram que os alienistas, em seu esforço para desenvolver um sistema descritivo, tendiam a optar por um dos termos cartesianos (fisiologia mecânica) em detrimento do outro (isto é, dos dados psicológicos) (D’Amiens, 1875). Estudos colaborativos foram tentados no início do século para preencher a lacuna, por exemplo, Antoine Royer-Collard convidou Maine de Biran para compor uma monografia sobre a relação entre a psicologia e a biologia (Royer-Collard, 1843; Maine de Biran, 1841; Swain, 1978). Um papel intermediário similar foi reivindicado para a psicopatologia durante o século XX (Deshaies, 1967; Thuilleaux, 1973).

\section{Psicopatologia e quantificação}

O fato de que no século XIX a psicopatologia descritiva tenha sido apenas marginalmente influenciada pela introdução da medição em medicina (Shryock, 1961) e nas ciências comportamentais (Boring, 1961; Zupan, 1976) exige explicação. As correlações normativas estabelecidas entre certas noções psicológicas associacionistas (por exemplo, "diferença apenas perceptível”; tempo de reação) e parâmetros físicos (Boring, 1942) nunca foram acompanhadas de estudos semelhantes com os doentes mentais (Ziehen, 1909; Whitwell, 1892).

Essa falha pode ter resultado do fato de que a maioria dos sintomas mórbidos reconhecidos pelos alienistas tiveram origem na psicologia das faculdades e constituíram "pacotes" de perturbação que atravessaram as unidades abstratas de análise dos associacionistas. Noções tais como confusão, estupor ou delírio eram muito complexas para a nova psicopatologia experimental ser capaz de manejar.

Além disso, qualquer desenvolvimento na "psicopatologia experimental" da doença mental iria sofrer um duro golpe próximo ao final do século pelo aparecimento dos modelos psicodinâmicos.

\section{A psicopatologia e a nova psicologia dinâmica}

Os modelos psicodinâmicos exploraram a distinção entre "forma e conteúdo" e fizeram disso seu mister ao desenvolver um instrumental conceitual para dar conta desse último. Descritivamente, no entanto, as teorias psicodinâmicas mantiveram-se surpreendentemente próximas à psicopatologia do século XIX, como mostra uma leitura atenta dos trabalhos precoces de Janet e Freud. A mudança gradual para "conteúdo" já havia ficado implícita nos trabalhos de Brentano (Fancher, 1977). Este autor, como Freud mais tarde, enfatizou a análise retrospectiva da experiência subjetiva (Ellenberger, 1970). A tradição do "hipnotismo" também contribuiu para a criação de um "espaço interno" ou "discurso" buscando os mecanismos não conscientes (Barrucard, 1967). Em Janet (Prevost, 


$\mid \begin{gathered}\text { HISTÓRIA DA } \\ \text { PSICOPATOLOGIA, } \\ \text { POR GERMAN E. } \\ \text { BERRIOS }\end{gathered}$

1973) e mais tarde em Freud (Bercherie, 1983) estas tendências convergiram e a nova "psicopatologia dinâmica" reuniu "conteúdo", "fonte" e "mecanismo" em um modelo teórico unificado.

\section{Contexto geral}

Linguagens (de vários graus de resolução) foram geradas pela maioria das sociedades e durante diversos períodos históricos para descrever a loucura, e seu estudo resultou na psicopatologia transcultural (Rack, 1982) e histórica, respectivamente. Períodos de estabilidade descritiva e de ruptura conceitual (Bachelard, 1938) podem ser discernidos na análise longitudinal. Cabe ao historiador identificar os mecanismos responsáveis por estes processos. Modelos de mudança científica (Hacking, 1981; Laudan, 1977; Pandit, 1983) podem ajudá-lo de um modo geral, mas ele necessita explicações regionais. O historiador clínico quer saber, por exemplo, como reconhecer os primeiros ou mais críticos sinais de uma "ruptura epistemológica" em sua própria disciplina. Mais importante, ele quer saber se essa crise decorre de falha de linguagem ou de mudança de objeto (Marchais, 1983). Este artigo afirma que a última "ruptura" na linguagem psicopatológica ocorreu durante a primeira metade do século XIX. Ele também sugere que o processo por meio do qual a nova psicopatologia foi construída foi primeiro concluído na França, logo após meados do século.

\section{O estado da arte histórica}

O Simpósio de Yale sobre a História da Psiquiatria ocorreu há 15 anos [escrito em 1984] (Mora \& Brand, 1970). Seus anais oferecem uma avaliação justa dos frutos da historiografia tradicional e são mais do que apenas "um livro informativo para neófitos" (Braceland, 1970). O mesmo espírito inovador levou Marx (1970) a afirmar em um trabalho clássico que para a escritura histórica nesta área era necessário "uma visão da história, uma definição de psiquiatria e um preceito do que a psiquiatria deve ser “ (p. 603); mais tarde ele ampliou essa visão lakatoshiana da história como um "constructo provisório" (Marx, 1977). Opiniões semelhantes foram expressas com relação à história das ciências comportamentais (Young, 1966) e à história da medicina (Clarke, 1971).

Desde aqueles dias felizes a história da psiquiatria tornou-se bem povoada. O esforço pós-foucaultiano não mostra sinais de diminuir, e as generalizações de seu mentor (Foucault, 1972b) foram submetidas a progressiva correção (Sedgwick, 1981; Castel, 1977; Swain, 1977; Gauchet \& Swain, 1980). Em uma 
corrente diferente, escritores franceses renomados, como Ey, Baruk, Lanteri-Laura e Postel publicaram, nos últimos anos, documentos históricos de primeira ordem que, infelizmente, permanecem praticamente desconhecidos no mundo anglófono.

Historiadores sociais também têm feito importantes contribuições para a história da psiquiatria (Scull, 1981; Alexander, 1976; Castel et al., 1979; Blasius, 1980; Dörner, 1969). Sua ênfase frequente sobre a aplicação da teoria dos controles sociais para a compreensão da loucura tem, por vezes, levado ao abandono de seus aspectos biológicos. Estas obras, portanto, podem ser vistas como menos relevantes para a compreensão da gênese da psicopatologia descritiva do que uma série de recentes publicações históricas (Werlinder, 1978; Janzarik, 1979; Wallace \& Pressley, 1980; Simon, 1978; Sauri, 1969; Roccatagliata, 1973, 1981; Lopez Piñero \& Morales Meseguer, 1970; Clarke, 1975; Dewhurst, 1982; Lopez Piñero, 1963; Bercherie, 1980; Postel \& Quétel, 1983).

Tendências historiográficas gerais também têm influenciado a história da psiquiatria (Brieger, 1980). Ocasionalmente, questões complexas na história das ciências comportamentais foram tornadas momentaneamente translúcidas pela aplicação fácil de termos kuhnianos tais como "paradigma", "anomalia", "comunidade científica", "mudança de Gestalt" (Kuhn, 1962). Assim, em vez de tentar desenvolver uma epistemologia das ciências inexatas (Helmer \& Rescher, 1959), alguns escritores se contentaram com a crença de que ciências do comportamento estariam descansando em algum tipo de "limbo pré-paradigmático" (Farrell, 1978; Warren, 1971). Um ponto de vista historiográfico anterior, e igualmente ingênuo, havia levado outros autores a considerar a psiquiatria pré-freudiana como "pré-histórica" (Zilboorg, 1941; Alexander \& Selesnick, 1966).

\section{A história e o clínico}

Parece haver, portanto, uma necessidade "legítima" (Daumezon, 1980) de estudos históricos que levantem questões específicas sobre a gênese das categorias descritivas psicopatológicas e sobre suas interações com o contexto psicológico e filosófico (Bercherie, 1980; Quétel \& Morel, 1979; Postel, 1981; Swain, 1977). Este artigo propõe um modelo de pesquisa que pode ser melhor descrito como um ninho de "caixas chinesas": com as caixas "interiores" contendo os conceitos filosófico-psicológicos, e as caixas "exteriores" o contexto sociopolítico. Afirma ainda que a análise conceitual da "caixa interior" já pode dar uma adequada conta da formação da linguagem psicopatológica. Esta "história conceitual" é particularmente adequada para a pesquisa em psicopatologia e fornece ao 


\begin{tabular}{c|c} 
HISTÓRIA DA \\
PSICOPATOLOGIA, \\
POR GERMAN E. \\
BERIOS \\
\hline
\end{tabular}

clínico técnicas práticas de calibração. A ênfase analítica nas "caixas interiores" não deve ser interpretada como uma rejeição das "caixas exteriores". Ela significa apenas que a análise histórica das últimas não é essencial para os efeitos práticos de compreensão da estrutura interna da psicopatologia descritiva.

\section{A hipótese histórica}

O modelo sentencial (Harré, 1970) proposto nesse trabalho pode ser resumido da seguinte forma (evidência detalhada pode ser encontrada em Berrios, 1988).

1. A psicopatologia descritiva desenvolveu-se durante a primeira metade do século XIX. O processo foi concluído primeiro na França.

2. Estabeleceu-se como uma linguagem descritiva. Como tal, ela continha uma terminologia, proposições relacionais e regras para sua aplicação.

3. O significado de cada termo passou a depender tanto da sua relação com uma determinada forma comportamental (função sinal) como de outros termos dentro do sistema descritivo (função de compatibilidade).

4. As regras para a aplicação de cada termo referem-se a: (a) avaliação do observador da intensidade, duração e qualidade da experiência dos sintomas tal como descritos pelo paciente, e (b) um número de associações permitidas, de acordo com o qual decisões sobre a presença de um sintoma eram tomadas em termos da presença ou ausência de outros sintomas, dentro de um determinado domínio.

5. A origem histórica da psicopatologia descritiva é multideterminada: fatores biológicos e sociais podem ser identificados.

6. Um fator social importante refere-se à exigência descritiva (a necessidade de manter bons registros clínicos), que os primeiros psiquiatras tiveram de se adequar. Para este fim, nomes genéricos (por exemplo, mania, melancolia, phrenitis) não eram suficientes. A fragmentação dessas categorias foi, portanto, necessária. Isso foi realizado em termos dos princípios da psicologia das faculdades mentais (Albrecht, 1970; Brooks, 1976) e do associacionismo (Warren, 1921; Hoeldtke, 1967), as duas influentes teorias psicológicas durante o início do século XIX na França (Boutroux, 1908; Ravaisson, 1885).

7. Um fator teórico importante foi representado por mudanças na epistemologia da medicina (Ackerknecht, 1967). O ponto de vista anatomoclínica, desenvolvido na França no início do século XIX (Lopez Piñero, 1983), exigiu que fossem estabelecidas correlações entre a lesão anatômica e as manifestações externas da disfunção, isto é, os sinais ou fenômenos (Laín Entralgo, 1978). 
8. O "descritivismo" que se seguiu logo se tornou uma "semiologia" (Barthes, 1972), isto é, um sistema cognitivo. Este novo sistema procurou legitimidade epistemológica em um retorno à visão baconiana de ciência (Ravaisson, 1885 , p. 60) como ilustrado pela reinterpretação positivista de Comte do datum baconiano (Rémusat, 1857). Relativo aos contatos diretos entre filósofos e alienistas, conta-se que em 1826 Comte fez palestras para um público selecionado que incluía Esquirol (Mazlish, 1967).

9. A fragmentação das categorias da loucura seguiu de perto a classificação tripartite das faculdades da mente que Kant (Hilgard, 1980) e os filósofos escoceses tinham reconstruído (Spoerl, 1936). Ao longo do tempo tanto distinções nosológicas quanto sintomáticas foram construídas ao longo destas linhas. Por exemplo, as alucinações (que originalmente se referiam apenas às experiências visuais) tornaram-se "distúrbios de percepção" em geral (Berrios, 1982) e a monomania (uma condição clínica) foi classificada em intelectual, emocional e volitiva (Linas, 1882).

10. Dois tipos de sintomas resultaram desta fragmentação. Alguns distúrbios como as alucinações poderiam ser considerados como exageros ou distúrbios da função normal (ponto de vista de continuidade). Outros estados que se referem ao bizarro, como as obsessões, os delírios ou o estupor, que não encontrariam nenhuma contrapartida clara no normal (ponto de vista da descontinuidade). Este sistema de duas camadas tem se mantido até nossa atualidade (Lanteri-Laura, 1968). O ponto de vista da continuidade pode ser encontrado nas obras de Freud (Broustra, 1975) e Eysenck (McGuire, 1973), e o ponto de vista da descontinuidade nos trabalhos de Jaspers (1963), Weibrecht (1968), Bash (1955) e Conrad (1958). Estas duas visões refletem de perto as permutações conceituais que ligavam o "normal" e o "patológico" durante o século XIX (Canguilhem, 1975).

11. No início do século XIX, a psicopatologia descritiva se baseava em dados obtidos a partir da observação de comportamento "manifesto". A utilização gradual de dados "psicológicos" ou "subjetivos" começou na França na década de 1840 (Dubois, 1845), principalmente com Moreau de Tours (Riese, 1960; Bollote, 1973; Pigeaud, 1980; Delacroix, 1924). Logo depois, a introdução das noções "psicológicas" de consciência (Berrios, 1981b) e de introspecção (Boring, 1953) legitimaram o valor semiológico dos "conteúdos de consciência". Este desenvolvimento histórico pode ajudar a compreender a origem da dicotomia "forma e conteúdo".

12. Um processo de seleção ocorreu após 1850 em que alguns sintomas foram retidos (delírios, por exemplo) (Ball \& Ritti, 1882) e outros foram descartados (por exemplo, hematoma aural e alucinações unilaterais) (Régis, 1881). O 


\begin{tabular}{c|c} 
HISTÓRIA DA \\
PSICOPATOLOGIA, \\
POR GERMAN E. \\
BERRIOS \\
\hline
\end{tabular}

mecanismo e a ideologia que presidiram esta seleção requerem maior elucidação. Apenas um pequeno número de sintomas sobreviveu de fato no século XX. Até o final do século XIX este número limitado de sintomas (e de permutações de sintomas) tinha se revelado insuficiente para fazer diagnósticos transversais confiáveis. Nosologistas fin de siècle como Magnan (Pichot, 1982) e Kraepelin (Havens, 1965) tentaram resolver o problema introduzindo fatores diagnósticos longitudinais ou "diacrônicos", tais como a irreversibilidade e a história natural. Em certas ocasiões, no entanto, isso conduziu a uma relativa negligência dos sintomas descritivos. Exemplificando, como Havens (1965, p. 23) observou, Kraepelin "raramente deu muita importância para os delírios diagnosticamente ou prognosticamente".

13. Na última parte do século XIX os sintomas sobreviventes foram reorganizados em algumas síndromes (Bercherie, 1980; Pichot, 1982). Alguns destes, como o delirium, permaneceram praticamente iguais a antes da fragmentação (Berrios, 1981a), outros, tais a insanidade maníaco-depressiva ou os transtornos obsessivos (Berrios, 1985), constituíram novas configurações. Alguns dos termos clássicos também foram reaproveitados (mania, melancolia, paranoia, demência e estupor), mas com um significado diferente (Leibbrand \& Wettley, 1961; Bercherie, 1980).

14. Logo no início do século XX, a psicopatologia descritiva encontrou-se com a fenomenologia. Esta muito falada aliança pode ser melhor descrita como um casamento de conveniência. Em torno deste período, a base conceitual da psicopatologia descritiva tinha se tornado suspeita (após o declínio da psicologia clássica do século XIX) e exigia escoramento. A Fenomenologia, com sua ênfase na subjetividade e descrições neutras, tornou-se a parceira ideal. Mas a Fenomenologia em si também se encontrava na necessidade de ancoragem empírica, o que resultava do fato de que, apesar de todo o seu antipsicologismo e exigências de neutralidade (De Boer, 1978), a fenomenologia se desenvolveu em uma outra variedade de psicologia de "poltrona" e ficou, portanto, teoricamente comprometida (Rancurello, 1968).

A eloquência juvenil de Jaspers (1968) fez parecer como se a fenomenologia tivesse sido posta, afinal, a trabalhar em um problema concreto; esta crença permanece até os dias de hoje (Shepherd, 1983; Spiegelberg, 1972).

Contudo, uma comparação cuidadosa do significado clínico e da utilização dos sintomas básicos, antes e depois de 1913 (Jaspers, 1963), mostra que o tratamento "fenomenológico" não os alterou em nada. De certa forma, isso não é surpreendente porque a utilização de Jaspers da "fenomenologia" neste estágio inicial era puramente metodológica: "Este trabalho preliminar de representar, definir e classificar fenômenos psíquicos, perseguido como uma atividade independente, constitui a fenomenologia" (Jaspers, 1968, p. 1314). 
Logo após, a fenomenologia iria se tornar um conglomerado de escolas (Spiegelberg, 1982). Embora algumas destas tenham se provado importantes para o desenvolvimento das psicologias e terapias existenciais (Spiegelberg, 1972), a fenomenologia era uma ferramenta metodológica muito abstrata e heterogênea para ter muita relevância para a psicopatologia descritiva prática. Subsequentes esforços neste sentido, tanto em psiquiatria (De Koning \& Jenner, 1982) como em psicologia clínica, têm sido bastante escassos (Merleau-Ponty, 1945; Thinès, 1977). De fato, a previsão de Ryle de que a fenomenologia iria "terminar afinal seja em subjetivismo autodestrutivo ou em um misticismo etéreo" parece ter se confirmado (Ryle, 1932).

\section{Conclusões}

Uma série de conclusões podem ser tiradas. Em primeiro lugar, estudos histórico-conceituais são parte integrante da construção da linguagem na psicopatologia pois, sem esses dados, a calibração estatística torna-se uma atividade incompleta. Em segundo lugar, a psicopatologia descritiva é uma atividade vinculada à linguagem que, apesar de sua crescente formalização científica, ainda continua dependente de uma tradição escrita e oral. Escolas e modas são mais relevantes para isso do que o clínico médio está disposto a admitir (Blashfield \& Feighner, 1982). Em terceiro lugar, é hora de que mais pesquisas sejam realizadas sobre a história dos sintomas individuais, idealmente, este trabalho deve ser realizado por médicos historicamente treinados e deve ser considerada como uma subespecialidade viável em psiquiatria. Em quarto lugar, atenção também deve ser dedicada à identificação da estrutura epistemológica da linguagem da psicopatologia. Neste contexto avanços mais importantes foram feitos na Europa (Daumezon, 1957; Daumezon \& Lanteri-Laura, 1961; Fédida, 1964; Pistoia, 1971; Castilla del Pino, 1978; Tizon, 1978; Georgin, 1980; Marchais, 1981; Lanteri-Laura, 1982, 1983) do que nos EUA (Kroll, 1979; McHugh \& Slavney, 1982).

Desde que instrumentos como o DSM-III (APA, 1980) e o PSE (Wing et al., 1974; Wing, 1983) estão sendo amplamente adotados como glossários, os clínicos em geral querem saber se os seus "direitos descritivos" em breve serão revogados por um "fechamento prematuro" (Spitzer et al. 1975, p. 1191), e, mais importante, eles querem saber se lhes sobra algum direito de tentar formas alternativas de psicopatologia (Shapiro, 1975; Clifford \& Frosh, 1982). Em face dos benefícios decorrentes da formalização estatística das descrições, perguntas como estas podem soar ingênuas ou desagradáveis. No entanto, vale ressaltar que um conhecimento mais familiarizado da história da psicopatologia descritiva pode moderar esforços agudos para alcançar confiabilidade e, quem sabe, lembrar a 


\begin{tabular}{c|c} 
HISTÓRIA DA \\
PSICOPATOLOGIA, \\
POR GERMAN E. \\
BERIOS \\
\hline
\end{tabular}

todos que a validade é também uma importante característica da linguagem científica. A história da psicopatologia deve ser considerada mais do que uma mera comédia de erros, exercício de antiquário, ou análise política dos aspectos sociais da psiquiatria. É uma poderosa técnica de calibração por meio da qual a linguagem da psiquiatria é conceitualmente ajustada e preparada para a quantificação.

\section{Referências}

ACKerknecht, E.H. Medicine at the Paris Hospital, 1794-1848. Baltimore: Johns Hopkins Press, 1967.

Albrecht, F.M. A reappraisal of faculty psychology. Journal of the History of the Behavioral Sciences, n. 6, p. 36-40, 1970.

Alexander, F.G.; Selesnick, S.T. The History of Psychiatry: An Evaluation of Psychiatric Thought and Practice from Pre-historic Times to the Present. New York: Harper, 1966.

Alexander, M.D. The administration of madness and attitudes towards the insane in nineteenth century Paris. Doctoral Dissertation. Baltimore: John Hopkins Press, 1976.

American Psychiatric Association. Diagnostic and Statistical Manual of Mental Disorders (DSM-III). 3rd. ed. Washington, D.C.: APA, 1980.

Andreasen, N. C. Should the term "Thought Disorder" be revised? Comprehensive Psychiatry, n. 23, p. 291-299, 1982.

Bachelard, G. La formation de l'esprit scientifique. Paris: Vrin, 1938.

Ball, B.; RitTi, A. Délire. In: Dictionnaire encyclopédique des sciences médicales. (ed. A. Dechambre). Paris: Asselin, 1882. V. 26, p. 315-408.

Barrucard, D. Histoire de l'hypnose en France. Paris: Presses Universitaires de France, 1967.

Barthes, R. Sémiologie et médecine. In: Les sciences de la folie. (ed. R. Bastide). Paris: Mouton, 1972. p. 37-46.

BASH, K. W. Lehrbuch der allegemeinen Psychopathologie. Grundbegriffe und Klinik. Stuttgart: Georg Thieme, 1955.

BERCHERIE, P. Les fondements de la clinique. Histoire et structure du savoir psychiatrique. Paris: La Bibliothèque d'Ornicar, 1980.

. Genèse des concepts freudiens. Paris: Navarin, 1983.

Berrios, G.E. Henri Ey, Jackson, et les idées obsédantes. L'évolution psychiatrique, n. 42 , p. 687-699, 1977. 
_ Stupor: a conceptual history. Psychological Medicine, n. 11, p. 677-688, 1981 a.

. Delirium and confusion in the 19th century: a conceptual history. British Journal of Psychiatry, n. 139, p. 439-499, 1981b.

. Tactile hallucinations: conceptual and historical aspects. Journal of Neurology, Neurosurgery and Psychiatry, n. 45, p. 285-293, 1982.

Orientation failures in medicine and psychiatry. Journal of the Royal Society of Medicine, n. 76, p. 249-256, 1983.

Obsessional Disorders during the Nineteenth Century: A Conceptual History. Terminological and Classificatory Issues. In: Bynum, W. F. et al (eds.). The Anatomy of Madness. London: Tavistock, 1985. V. 1, p. 166-187.

Historical background to abnormal Psychology. In: Miller, E.; CoOper, P. (eds.). Adult Abnormal Psychology. London: Churchill Livingstone, 1988. p. 26-51.

Blashfield, R.K.; Feighner, J.P. Invisible colleges and the Matthew effect. Schizophrenia Bulletin, n. 8, p. 1-6, 1982.

Blasius, D. Der verwaltete Wahnsinn. Eine Sozialgeschichte des Irrenhauses. Frankfurt: Fischer Taschenbuch, 1980.

Bollote, G. Moreau de Tours 1804-1884. Confrontations psychiatriques, n. 11, p. 9$-26,1973$.

Boring, E.G. Sensation and Perception in the History of Experimental Psychology. New York: Appleton-Century-Crofts, 1942.

. A history of introspection. Psychological Bulletin, n. 50, p. 169-189, 1953.

. The beginning and growth of measurement in psychology. Isis, n. 52, p. 238$-257,1961$.

Boutroux, E. De l'influence de la philosophie écossaise sur la philosophie française. In: Études d'histoire de la philosophie (ed. E. Boutroux). Paris: Alcan, 1908. p. 413-433.

Braceland, F.J. Foreword. In: Psychiatry and its History. Methodological Problems in Research (ed. G. Mora and J.L. Brand). Springfield. Ill.: Charles Thomas, 1970. p. vii.

BRIEGER, G.H. History of medicine. In A Guide to the Culture of Science. Technology and Medicine (ed. P. T. Durbin). New York: Free Press, 1980. p. 121-194.

BRooks, G.P. The faculty psychology of Thomas Reid. Journal of the History of the Behavioral Sciences, n. 12, p. 65-77, 1976.

Broustra, J. Les schizophrènes. Paris: Jean-Pierre Delarge, 1975. Cap. 5.

BurcKard, E. Les conceptions psychiatriques de Wernicke. Travaux de la clinique psychiatrique de Strassbourg, n. 9, p. 45-141, 1931.

Canguilhem, G. Le normal et le pathologique. 3rd ed. Paris: Presses Universitaires de France, 1975.

Rev. Latinoam. Psicopat. Fund., São Paulo, v. 15, n. 1, p. 171-196, março 2012 


\begin{tabular}{c|c} 
HISTÓRIA DA \\
PSICOPATOLOGIA, \\
POR GERMAN E. \\
BERIOS \\
\hline
\end{tabular}

Carpenter, W.B. Principles of Mental Physiology. London: C. Kegan Paul \& Co., 1879.

CASTEL, R. Le psychanalysme. L'ordre psychanalytique et le pouvoir. Paris:

Françoise Maspero, 1973.

. L'ordre psychiatrique. L'âge d'or de l'aliénisme. Paris: Minuit, 1977.

Castel, R., Castel, F.; Lovell, A. La societé psychiatrique avancée. Paris: Grasset et Fasquelle, 1979.

Castilla del Pino, C. Introducción a la psiquiatria - Vol. 1: Problemas Génerales, Psico(pato)logia. Madrid: Alianza Editorial, 1978.

Clarke, B. Mental Disorders in Earlier Britain: Exploratory Studies. Cardiff:

University of Wales Press, 1975.

Clarke, E. (ed.). Modern Methods in the History of Medicine. London: Athlone Press, 1971.

Clifford, P.; Frosh, S. Towards a non-essentialist psychology: a linguistic

framework. Bulletin of the British Psychological Society, n. 35, p. 267-270, 1982.

Condillac, E.B. Traité de systèmes. In: Oeuvres philosophiques de Condillac. (ed. G. Le Roy). Paris: Presses Universitaires de France, 1947. V. 1, p. 121-217.

CONRAD, K. Die beginnende Schizophrenie. Versuch einer Gestalanalyse des Wahns. Stuttgart: Georg Thieme, 1958.

D’Amiens, D. Folie. In: Dictionnaire des sciences philosophiques par une société de professeurs et de savants (ed. A. D. Franck). Paris: Hachette, 1875. p. 548-552.

Daumezon, G. Reflexions sur la semiologie psychiatrique. L'évolution psychiatrique, n. 22, p. 207-237, 1957.

. Légitimité de l'intêret pour l'histoire de la psychiatrie. L'information psychiatrique, n. 65, p. 647-653, 1980.

Daumezon, G.; Lanteri-Laura, G. Signification d'une semiologie. L'Encephale, n. 14, p. 478-511, 1961.

De Boer, T. The Development of Husserl's Thought. Nijhoff: The Hague, 1978.

De Koning, A.J.J.; Jenner, F.A. (eds.). Phenomenology and Psychiatry. London: Academic Press, 1982.

Delacroix, M. H. Maine de Biran et l'école médico-psychologique. Bulletin de la Société françoise de philosophie, n. 24, p. 51-63, 1924.

Deshaies, G. Psychopathologie générale. Paris: Presses Universitaires de France, 1967.

Dewhurst, K. Hughlings Jackson on Psychiatry. Oxford: Sandford Publications, 1982. 
DöRnER, K. Bürger und Irre. Zur Sozialgeschichte und Wissenschaftsoziologie der Psychiatrie. Frankfurt: Europaische Verlagsanstalt, 1969.

Dubors, F. Quelques considérations sur l'aliénation mentale au point de vue de la psychologie. Bulletin de l'Académie royale de médecine, n. 10, p. 533-543, 1845.

Ellenberger, H. The Discovery of the Unconscious. The History and Evolution of Dynamic Psychopathology. London: Allen Lane, 1970.

FALRET, J.F. Des maladies mentales et des asiles d'alienés. Paris: J. B. Baillière, 1864.

FANCHER, R.E. Brentano's psychology from an empirical standpoint and Freud's early metapsychology. Journal of the History of the Behavioral Sciences, n. 13, p. 207-227, 1977.

Farrell, B.A. The progress of psychology. British Journal of Psychology, n. 69, p. 1-8, 1978.

FÉDIDA, P. Le structuralisme en psychopathologie. L'évolution psychiatrique, n. 29, p. 85-129, 1964.

FLECK, L. Genesis and Development of a Scientific Fact. Chicago: University of Chicago Press, 1979.

Foucault, M. Naissance de la clinique, 2rd ed. Paris: Presses Universitaires de France, 1972a.

. Histoire de la folie a l'âge classique. Paris: Gallimard, 1972b.

FRANCK, A.D. Système. In: Dictionnaire des sciences philosophiques par une société de professeurs et de savants. 2rd ed. (ed. A. D. Franck). Paris: Hachette, 1875a. p. 1703-1704.

. Signes. In: Dictionnaire des sciences philosophiques par une société de professeurs et de savants. 2rd ed. (ed. A. D. Franck). Paris: Hachette, 1875b. p. 16081616.

Gauchet, M.; Swain, G. La pratique de l'esprit humain. Paris: Gallimard, 1980.

Georgin, B. Remarques sur le discours nosologique en psychiatrie. L'évolution psychiatrique, n. 45, p. 5-17, 1980.

Griesinger, W. Mental Pathology and Therapeutics. Transl. C. L. Robertson and J. Rutherford. London: New Sydenham Society, 1867.

. Vortrag zur Eröffnung der psychiatrischen Klinik zu Berlin. In: Gesammelte Abhandlungen. Berlin: Hirschwald, 1875. V. I, p. 127-129.

Hacking, I. (ed.). Scientific Revolutions. Oxford: Oxford University Press, 1981.

Hare, E.H. The changing content of psychiatric illness. Journal of Psychosomatic Research, n. 18, p. 283-289, 1974.

Rev. Latinoam. Psicopat. Fund., São Paulo, v. 15, n. 1, p. 171-196, março 2012 


\begin{tabular}{c|c} 
HISTÓRIA DA \\
PSICOPATOLOGIA, \\
POR GERMAN E. \\
BERIOS \\
\hline
\end{tabular}

Harré, R. The Principles of Scientific Thinking. London: MacMillan, 1970. p. 33.

Havens, L. Emil Kraepelin. Journal of Nervous and Mental Disease, n. 141, p. 16-28, 1965.

Helmer, O.; Rescher, N. On the epistemology of the inexact sciences. Management Science, n. 8, p. 25-52, 1959.

Hesse, M.B. Models and Analogies in Science. Paris: University of Notre Dame Press, 1966.

Hilgard, E.R. The trilogy of mind: cognition, affection, and conation. Journal of the History of the Behavioral Sciences, n.16, p. 107-117, 1980.

HoEldtKe, R. The history of associationism and British medical psychology. Medical History, n. 11, p. 46-64, 1967.

Holton, G. Thematic Origins of Scientific Thought. Cambridge, Mass.: Harvard University Press, 1973.

JANZARIK, W. (ed.). Psychopathologie als Grundlagenwissenschaft. Stuttgart: Enke, 1979.

JASPERS, K. General Psychopathology. Transl. M. Hamilton and J. Hoenig.

Manchester: Manchester University Press, 1963. . The phenomenological approach in psychopathology. British Journal of Psychiatry, n. 114, p. 1313-1323, 1968.

Kendler, K.S.; Tsuang, M.T. Nosology of paranoid schizophrenia and other paranoid psychoses. Schizophrenia Bulletin, n. 7, p. 594-610, 1981.

KROLL, J. Philosophical foundations of French and US nosology. American Journal of Psychiatry, n. 136, p. 1135-1138, 1979.

Kunn, T. The Structure of Scientific Revolutions. Chicago: Chicago University Press, 1962.

Laín Entralgo, P. Historia de la medicina. Barcelona: Salvat, 1978.

LANTERI-Laura, G. Psychologie pathologique. In: Encyclopédie médico-chirurgicale. Psychiatrie. Paris: Editions Techniques, 1968. V. I. $37032 \mathrm{C}^{10}$.

. La connaissance clinique: histoire et structure en médecine et en psychiatrie. L'évolution psychiatrique, n. 47, p. 423-469, 1982.

. La sémiologie psychiatrique: son évolution et son état en 1982. L'évolution psychiatrique, n. 48, p. 327-363, 1983.

Laudan, L. Progress and its Problems. Towards a Theory of Scientific Growth. Berkeley: University of California Press, 1977.

Le Roy, G. La psychologie de Condillac. Paris: Boivin et Cie., 1937.

LeibBrand, W.; Wettley, A. Der Wahnsinn. Geschichte der abendländischen Psychopathologie. Freiburg: Karl Alber, 1961.

Rev. Latinoam. Psicopat. Fund., São Paulo, v. 15, n. 1, p. 171-196, março 2012 
$\begin{array}{lllllll}R & E & V & \text { I } & S & T & A\end{array}$

LATINOAMERICANA

DE PSICOPATOLOGIA

F U N D A M N T A L

LeOnhard, K. Aufteilung der endogenen Psychosen. Berlin: Akademie, 1957.

Linas, A. Monomanie. In: Dictionnaire encyclopédique des sciences médicales. 5th Vol., 2nd Series. (ed. A. Dechambre). Paris: Asselin, 1882. p. 146-195.

Lopez Piñero, J.M. Origenes históricos del concepto de neurosis. Valencia: Instituto de Historia de la Medicina, 1963.

Historical Origins of the Concept of Neurosis. Transl. D. Berrios. Cambridge: Cambridge University Press, 1983.

Lopez Piñero, J.M.; Morales Meseguer, J.M. Neurosis y psicoterapia. Un estudio histórico. Madrid: Espasa-Calpe, 1970.

Mackenzie, T.B.; Rosenberg, S.D.; Bergen, B.L. The manipulative patient: an interaction approach. Psychiatry, n. 41, p. 264-271, 1978.

Maine de BiRAn. Nouvelles considérations sur les rapports du physique et du moral de l'homme. In: Oeuvres philosophiques de Maine de Biran. (ed. V. Cousin). Paris: Librairie de Ladrange, 1841. V. 4, p. 1-146.

Marchais, P. La méthode systèmale en psychiatrie. Annales médico-psychologiques, n. 135, p. 677-695, 1977. . Les processus psychopathologiques de l'adulte. Paris: Privat, 1981.

Érès, 1983.

Les mouvances psychopathologiques: essai de psychiatrie dynamique. Paris:

MARx, O.M. What is the history of psychiatry? American Journal of Orthopsychiatry, n. 40, p. 593-605, 1970.

. History of psychology: a review of the last decade. Journal of the History of the Behavioral Sciences, n. 13, p. 41-77, 1977.

Mazlish, B. Comte, Auguste. In: The Encyclopedia of Philosophy. (ed. P. Edwards). New York: Macmillan, 1967. V. 2, p. 173-177.

McGuiRe, R. Classification and the problem of diagnosis. In: Handbook of Abnormal Psychology. (ed. H. J. Eysenck). London: Pitman Medical, 1973. p. 3-33.

McHugh, P.R.; Slavney, P.R. Methods of reasoning in psychopathology: conflict and resolution. Comprehensive Psychiatry, n. 23, p. 197-215, 1982.

McLeod, R. Changing perspectives in the history of science. In: Science, Technology and Society: A Cross Disciplinarian Perspective. (ed. I. Spiegel-Rosing and D.D.S. Price). London: Sage Publications, 1977. p. 149-196.

Merleau-Ponty, M. Phénoménologie de la perception. Paris: Gallimard, 1945.

Miller, E. \& Berrios, G. E. Medical model and psychiatry, 1984. (Submitted for publication.)

Rev. Latinoam. Psicopat. Fund., São Paulo, v. 15, n. 1, p. 171-196, março 2012 


\begin{tabular}{c|c} 
HISTÓRIA DA \\
PSICOPATOLOGIA, \\
POR GERMAN E. \\
BERIOS \\
\hline
\end{tabular}

Mora, G.; Brand, J.L. (eds.). Psychiatry and its History. Methodological Problems in Research. Springfield, Ill.: Charles Thomas, 1970.

Munro, A. Paranoia revisited. British Journal of Psychiatry, n. 141, p. 344-349, 1982.

Neve, M. Up market in Manhattan. Times Literary Supplement, n. 4170, p. 208, 1983.

Pandit, GL. The Structure and Growth of Scientific Knowledge. Dordrecht: Reidel, 1983.

Parain-Vial, J. La nature du fait dans les sciences humaines. Paris: Presses

Universitaires de France, 1966.

Ріснот, P. The diagnosis and classification of mental disorders in French-speaking countries: background, current views and comparison with other nomenclatures. Psychological Medicine, n. 12, p. 475-492, 1982.

Pigeaud, J.M. Le rôle des passions dans la pensée médicale de Pinel à Moreau de Tours. History and Philosophy of the Life Sciences, n. 2, p. 123-140, 1980.

Pistoia, L. del. Le problème de la temporalité dans la psychiatrie française classique. L'évolution psychiatrique, n. 36, p. 445-474, 1971.

Postel, J. Genèse de la psychiatrie. Les premiers écrits de Philippe Pinel. Paris: Le Sycamore, 1981.

Postel, J.; Quétel, C. (eds.). Nouvelle histoire de la psychiatrie. Paris: Privat, 1983.

Prevost, C.M. La psycho-philosophie de Pierre Janet. Paris: Payot, 1973.

QuÉTEL, C.; Morel, P. Les fous et leurs médecins de la renaissance au XXe siècle. Paris: Hachette, 1979.

RAck, P. Race, Culture and Mental Disorder. London: Tavistock Publications, 1982.

Rancurello, A.C. A Study of Franz Brentano, His Psychological Standpoint and his Significance in the History of Psychology. New York: Academic Press, 1968.

Ravaisson, F. (1867). La philosophie en France au XIX ${ }^{e}$ siècle. 2nd ed.). Paris: Hachette, 1885.

RÉGIS, E. Des hallucinations unilatéerales. L'Encéphale, n. 1, p. 43-74, 1881.

RÉmusat, C. de. Bacon, sa vie, son temps, sa philosophie, et son influence jusqu'à nos jours. Paris: Hachette, 1857.

Rescher, N. Cognitive Systematization. A System-theoretic Approach to a Coherentist Theory of Knowledge. Oxford: Basil Blackwell, 1979.

RIESE, W. The impact of nineteenth century thought on psychiatry. International Record of Medicine, n. 173, p. 7-19, 1960.

. La méthode analytique de Condillac et ses rapports avec l'oeuvre de Philippe Pinel. Revue Philosophique, n. 158, p. 321-336, 1968. 
$\begin{array}{llllllll}R & E & V & \text { I } & S & T & A\end{array}$

LATINOAMERICANA

DE PSICOPATOLOGIA

F U N D A M E T A L

Roccatagliata, G. Storia della psichiatria Antica. Milan: Ulrico Hoepli, 1973. . Storia della psichiatria biologica. Florence: Nuova Guaraldi, 1981.

Rome, H.P. The classification of schizophrenia: a historical review. Psychiatric Annals, n. 9, p. 5-13, 1979.

Royer-Collard, H. Examen de la doctrine de Maine de Biran sur les rapports du physique et du moral de l'homme par Antoine-Athanase Royer-Collard. Annales médico-psychologiques, n. 2, p. 9-45, 1843.

Ryle, G. Phenomenology. Proceedings of the Aristotle Society, n. 11 (Suppl.), p. 68$-83,1932$.

SAURI, J.J. Historia de las ideas psiquiatricas. Buenos Aires: Carlos Lohlé, 1969.

Scull, A. T. Museums of Madness. The Social Organization of Insanity in Nineteenth Century England. London: Allen Lane, 1979.

Scull, A. T. (ed.). Madhouses, Mad-doctors and Madmen. The Social History of Psychiatry in the Victorian Era. London: Athlone Press, 1981.

Sedgwick, P. Michel Foucault: The anti-history of psychiatry. Psychological Medicine, n. II, p. 235-248, 1981.

ShapIRo, M.B. The requirements and implications of a systematic science of psychopathology. Bulletin of the British Psychological Society, n. 28, p. 149-155, 1975.

SHEPHERD, M. Introduction: The sciences and general psychopathology. In:

Handbook of Psychiatry - Vol. I: General Psychopathology. (ed. M. Shepherd and O. L. Zangwill). Cambridge: Cambridge University Press, 1983. p. 1-8.

SHRYOCK, R.H. The history of quantification in medical science. Isis, n. 52, p. 215-237, 1961.

Simon, B. Mind and Madness in Ancient Greece. The Classical Roots of Modern Psychiatry. Ithaca: Cornell University Press, 1978.

Spiegelberg, H. Phenomenology in Psychology and Psychiatry. Evanston:

Northwestern University Press, 1972. . The Phenomenological Movement. A Historical Introduction. Nijhoff: The Hague, 1982.

SPITZER, R.L.; Endicott, J.; Robins, E. Clinical criteria for psychiatric diagnosis and DSM-III. American Journal of Psychiatry, n. 132, p. 1187-1192, 1975.

Spoerl, H.D. Faculties versus traits: Gall's solution. Character and Personality, n. 4, p. 216-231, 1936.

StARobinski, J. The role of language in psychiatric treatment in the French Romantic Age. A note on Dr. Laurent Cerise. Psychological Medicine, n. 4, p. 360-363, 1974. 


\begin{tabular}{c|c} 
HISTÓRIA DA \\
PSICOPATOLOGIA, \\
POR GERMAN E. \\
BERRIOS \\
\hline
\end{tabular}

SÜlz, K.D.; Gigerenzer, G. Über die Beeinflussung psychiatrischer Diagnoseschemata durch implizite nosologische Theorien. Archiv für Psychiatrie und Nervenkrankheiten, n. 232, p. 5-14, 1982.

Swain, G. Le sujet de la folie. Naissance de la psychiatrie. Paris: Privat, 1977. . L'aliéné entre le médecin et le philosophe. Perspective psychiatriques, n. 65, p. 90-99, 1978.

THinès, G. Phenomenology and the Science of Behaviour. London: George Allen and Unwin, 1977.

Thuilleaux, M. Connaissance de la folie. Paris: Presses Universitaires de France, 1973.

Tizon, J.L. Introducción a la epistemologia de la psicopatologia y la psiquiatria. Barcelona: Ariel, 1978.

Wallace, E.R.IV.; Pressley, L.C. Essays on the History of Psychiatry. South Carolina: Hall Psychiatric Institute, 1980.

Warren, H.C. History of the Association Psychology. New York: Scribner's Sons, 1921.

WARrEn, N. Is a scientific revolution taking place in psychology? Scientific Studies, n. 1, p. 407-413, 1971.

Wartofsky, M.W. Models. Representation and the Scientific Understanding. Dordrecht: Reidel, 1979.

Weibrecht, H. J. Psychiatrie im Grundriss. 2nd ed. Berlin: Springer, 1968.

Werlinder, H. Psychopathy: A History of the Concepts. Analysis of the Origin and Development of a Family of Concepts in Psychopathology. Acta Universitatis Upsaliensis: Upsala, 1978.

Whitwell, J.R. Pulse in insanity. In: A Dictionary of Psychological Medicine. (ed. D. H. Tuke). London: Churchill, 1892. 2 vols., p. 1042-1052.

WING, J. K. Use and misuse of the PSE. British Journal of Psychiatry, n. 143, p. 111$-117,1983$.

Wing, J.K.; CoOper, J.E.; SARTorius, N. Measurement and Classification of Psychiatric Symptoms. Cambridge: Cambridge University Press, 1974.

Young, R.M. Scholarship and the history of the behavioural sciences. History of Science, n. 5, p. 1-51, 1966.

ZIEHEN, T. Introduction to Physiological Psychology. Transl. C. C. Van Liew and O. W. Bryer. London: Swan Sonnenschein, 1909.

ZILboorg, G. A History of Medical Psychology. New York: Norton, 1941. 
ZuPAN, M.L. The conceptual development of quantification in experimental psychology. Journal of the History of the Behavioral Sciences, n. 12, p. 145-158, 1976.

\section{Resumos}

(Descriptive Psychopathology: Historical and Conceptual Aspects)

This article consists of a historical and conceptual analysis of descriptive psychopathology. The first section defines this field as a cognitive system consisting of terms, suppositions and rules for application. The section conceptual implications of this definition are outlined and related to clinical practice. The second section consists of an updated evaluation of the historical work carried out in descriptive psychopathology and presents a new hypothesis to explain how it developed during the 19th century. The author suggests that the testing of hypotheses should be conducted by psychiatrists with training in history, and seen as a separate specialty. We conclude that investigation into the history of the psychiatric symptoms of patients should be considered an essential stage in the construction of a feasible descriptive psychopathology.

Key words: Psychopathology - history, psychological models, psychiatry - history, language

(Psychopathologie descriptive: aspects historiques et conceptuels)

Cet article propose une analyse conceptuelle et historique de la psychopathologie descriptive. La première section la définit comme un système cognitif composé de termes, de suppositions et de règles pour son application. Elle expose les implications conceptuelles de cette définition et les relie à la pratique clinique. La deuxième section contient une évaluation de l'ouvrage historique sur la psychopathologie descriptive et offre une hypothèse qui explique son développement au cours du XIXe siècle. L'article suggère que le travail impliqué dans le test des hypothèses doit être effectué par des psychiatres qualifié en histoire et en tant que spécialité distincte. Il s'ensuit que l'histoire de l'étalonnage des symptômes psychiatriques doit être considérée comme une étape essentielle de la construction d'une psychopathologie descriptive viable.

Mots clés: Psychopathologie - histoire, modèles psychologiques; psychiatrie - histoire, langage

(Psicopatologia Descriptiva: aspectos históricos y conceptuales)

Este artículo ofrece um anális conceptual e histórico de la psicopatologia descriptiva. En La primera parte se la define como um sistema cognitivo constituido por términos, suposiciones y reglas para su aplicación. Se delinea las implicaciones 


\begin{tabular}{c|c} 
HISTÓRIA DA \\
PSICOPATOLOGIA, \\
POR GERMAN E. \\
BERIOS \\
\hline
\end{tabular}

conceptuales de esa definición y se las relaciona con la práctica clínica. La segunda parte contiene una evaluación del trabajo histórico sobre la psicopatologia descriptiva y ofrece una hipótesis para explicar su desarrollo durante el siglo XIX. Se sugiere que el trabajo supuesto en el testeo de hipótesis debe ser realizado por psiquiatras con formación en historia como una especialidad distinta. Se concluye que calibrar históricamente los síntomas psiquiátricos debe ser considerado como una etapa esencial en la construcción de una psicopatologia descriptíva viable.

Palabras clave: Psicopatologia - historia, modelos psicológicos, psiquiatria -historia, lenguaje

(Deskriptive Psychopathologie: historische und konzeptionelle Aspekte)

Dieser Beitrag bietet eine konzeptionelle und historische Untersuchung der deskriptiven Psychopathologie. Im ersten Teil wird sie als ein kognitives System aus Begriffen, Annahmen und Anwendungsregeln beschrieben. Es werden die konzeptionellen Implikationen dieser Definition aufgeführt und der klinischen Praxis gegenübergestellt. Der zweite Teil umfasst eine Zusammenfassung der historischen Arbeit bezüglich der deskriptiven Psychopathologie und präsentiert eine Hypothese zur Erklärung ihrer Entwicklung im 19. Jahrhundert. Es wird vorgeschlagen, dass die Aufgaben des HypotheseTests von Psychiatern mit einer Ausbildung in Geschichte und als eigenständige Spezialisierung durchgeführt werden sollten. Man ist zu dem Schluss gekommen, dass die historische angemessene Einschätzung der psychiatrischen Symptome eine wesentliche Etappe in der Entwicklung einer umsetzbaren deskriptiven Psychopathologie sein sollte.

Schlüsselwörter: Psychopathologie - Geschichte, psychologische Modelle, Psychiatrie Geschichte, Sprache

Citação/Citation: Berrios, G.E. Psicopatologia descritiva: aspectos históricos e conceituais. Revista Latinoamericana de Psicopatologia Fundamental, São Paulo, v. 15, n. 1, p. 171-196, março 2012.

Editor do artigo/Editor: Prof. Dr. Manoel Tosta Berlinck

Rev. Latinoam. Psicopat. Fund., São Paulo, v. 15, n. 1, p. 171-196, março 2012 


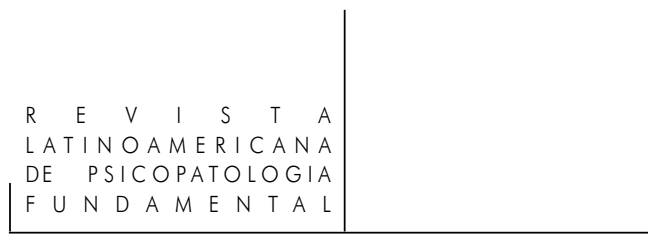

Recebido/Received: 23.4.2011 / 4.23.2011 Aceito/Accepted: 25.7.2011 / 7.25.2011

Copyright: () 2009 Associação Universitária de Pesquisa em Psicopatologia Fundamental/ University Association for Research in Fundamental Psychopathology. Este é um artigo de livre acesso, que permite uso irrestrito, distribuição e reprodução em qualquer meio, desde que o autor e a fonte sejam citados/This is an open-access article, which permits unrestricted use, distribution, and reproduction in any medium, provided the original author and source are credited.

Financiamento/Funding: $\mathrm{O}$ autor declara não ter sido financiado ou apoiado/The author has no support or funding to report.

Conflito de interesses/Conflict of interest: $O$ autor declara que não há conflito de interesses/The author declares that has no conflict of interest.

\section{German E. Berrios}

Médico e filósofo pela Universidad Nacional de San Marcos, Lima, Peru; Psiquiatra; Neurologista; Psicólogo; Filósofo; Historiador e Filósofo da ciência (Oxford University, England); Professor de Neuropsiquiatria e de Epistemologia da Psiquiatria (University of Cambridge, England), desde 1976; Neuropsiquiatra e Chefe do Departamento de Neuropsiquiatria do Hospital Addenbrooke, University of Cambridge, por 32 anos; Coordenador do Comitê de Ética em Pesquisa com Humanos na mesma universidade, por 20 anos; Editor Responsável de History of Psychiatry; Autor de 14 livros, incluindo The History of Mental Symptoms, Descriptive Psychopathology since 19th Century (Prêmio Nacional BMA, 1997), A History of Clinical Psychiatry (com Roy Porter), e Delirio (com F. Fuentenebro) e mais de 400 artigos e capítulos de livros; Membro do Royal College of Psychiatrists; da Associação Britânica de Psicologia e da Academia Britânica de Ciências Médicas; Membro Vitalício do Robinson College, Cambridge; doutor Honoris-Causa da Universidade de Heidelberg (Alemanha), da Universidade Nacional Mayor de San Marcos (Peru) e da Universidad Autónoma de Barcelona (Espanha); Grão Oficial da Ordem del Sol (Condecoração do Governo Peruano, 2007); prêmio Ramon y Cajal 2008 concedido pela Asociación Internacional de Neuropsiquiatria.

University of Cambridge

Box 189, Hills Road

Cambridge,

UK CB2 2QQ

e-mail: geb11@cam.ac.uk 